\title{
openheart Adjunctive interpulmonary isthmus ablation has no added effects on atrial fibrillation recurrence
}

\author{
Shunichi Higashiya, ${ }^{1}$ Hirosuke Yamaji, ${ }^{1}$ Takashi Murakami, ${ }^{1}$ Kazuyoshi Hina, ${ }^{1}$ \\ Hiroshi Kawamura, ${ }^{1}$ Masaaki Murakami, ${ }^{1}$ Shigeshi Kamikawa, ${ }^{1}$ Issei Komatsubara, ${ }^{2}$ \\ Shozo Kusachi ${ }^{1}$
}

To cite: Higashiya S, Yamaji H Murakami T, et al. Adjunctive interpulmonary isthmus ablation has no added effects on atrial fibrillation recurrence. Open Heart 2017:4:e000593. doi:10.1136/ openhrt-2017-000593

Received 11 January 2017 Revised 22 February 2017 Accepted 28 February 2017

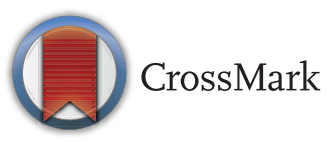

'Heart Rhythm Center, Okayama Heart Clinic, Okayama, Japan ${ }^{2}$ Department of General Internal Medicine, Kawasaki Hospital, Kawasaki Medical School, Okayama, Japan

Correspondence to Dr Hirosuke Yamaji; yamaji2@ mac.com

\section{ABSTRACT}

Objective Data on the efficacy of adjunctive interpulmonary isthmus ablation following completion of extensive encircling pulmonary vein isolation (EEPVI) on atrial fibrillation (AF) recurrence have still been insufficient. We aimed to compare the AF recurrence between EEPVI with and without adjunctive interpulmonary isthmus ablation.

Methods We enrolled 200 consecutive patients with paroxysmal AF (first session) who underwent EEPVI with double-Lasso technique. Patients were prospectively randomised into two groups: EEPVI with (group 1) and without (group 2) adjunctive interpulmonary isthmus ablation.

Results No differences were found in patients' clinical and echocardiographic backgrounds, including arrhythmia status, between the two groups. No differences were also observed in complications (two groin haematoma in both groups). All patients in both groups reached the EEPVI endpoint. The AF recurrence rate between groups $1(32 / 100,32 \%)$ and $2(33 / 100$, $33 \% ; p=1.0$ ) was quite similar during the follow-up period (45 \pm 5 months; $36-54$ months). The two groups showed identical Kaplan-Meier AF-free curves ( $p=0.460$; NS). Similar pulmonary vein (PV) reconnection incidence was observed in both groups during the second session. Durable isolation between the superior and inferior PVs was confirmed in $88 \%(21 / 27)$ of patients in group 1 , indicating that interpulmonary isthmus ablation maintained a non-conducting state in a considerable number of patients. Nevertheless, AF recurrence was identical between the two groups.

Conclusion The results of our study showed similar AF recurrence rates between the two groups, indicating that adjunctive interpulmonary isthmus ablation with EEPVI has no obvious effects on AF recurrence.

\section{INTRODUCTION}

Radiofrequency catheter ablation has currently become the most effective treatment for symptomatic drug-refractory atrial fibrillation (AF). ${ }^{12}$ Pulmonary veins (PVs) are the main trigger source that induce $\mathrm{AF}$. In addition, interpulmonary isthmus zone, which is in the carina region, as well as superior vena cava, coronary sinus musculature, Marshall vein, left atrium (LA) posterior

\section{KEY QUESTIONS}

What is already known about this subject?

- Extensive encircling pulmonary vein isolation (EEPVI) is currently a standard method to treat atrial fibrillation (AF). AF recurrence is a major problem to be resolved. Data on the efficacy of adjunctive interpulmonary isthmus ablation following completion of EEPVI on AF recurrence have still been insufficient.

What does this study add?

- We compared the AF recurrence rate between EEPVI by using the double-Lasso technique with $(n=100)$ and without $(n=100)$ adjunctive interpulmonary isthmus ablation in patients with paroxysmal AF. The AF recurrence rate was similar between EEPVI with (32\%) and without (33\%) interpulmonary isthmus ablation. The Kaplan-Meier AF-free curves were identical for the two groups.

How might this impact on clinical practice?

- The results showed that adjunctive interpulmonary isthmus ablation with EEPVI has no significant additive effects on AF recurrence. Consequently, adjunctive interpulmonary isthmus ablation with EEPVI is not recommended in AF ablation to reduce the recurrence rate.

wall and left atrial appendage are capable of inducing $\mathrm{AF}^{4}$

With the development of ablation systems and technology, and devices including catheters, success rates without antiarrhythmic drug use and overall success rates are currently approximately $75 \%$ in paroxysmal $\mathrm{AF}^{5}$ However, the remaining patients are still associated with $\mathrm{AF}$ recurrence.

Extensive encircling pulmonary vein isolation (EEPVI) is currently a widely applied treatment method for AF. Previous studies have demonstrated the presence of electrical connections between ipsilateral PVs. ${ }^{6}$ The interpulmonary isthmus region has also been proven to be a PV trigger origin initiating and 

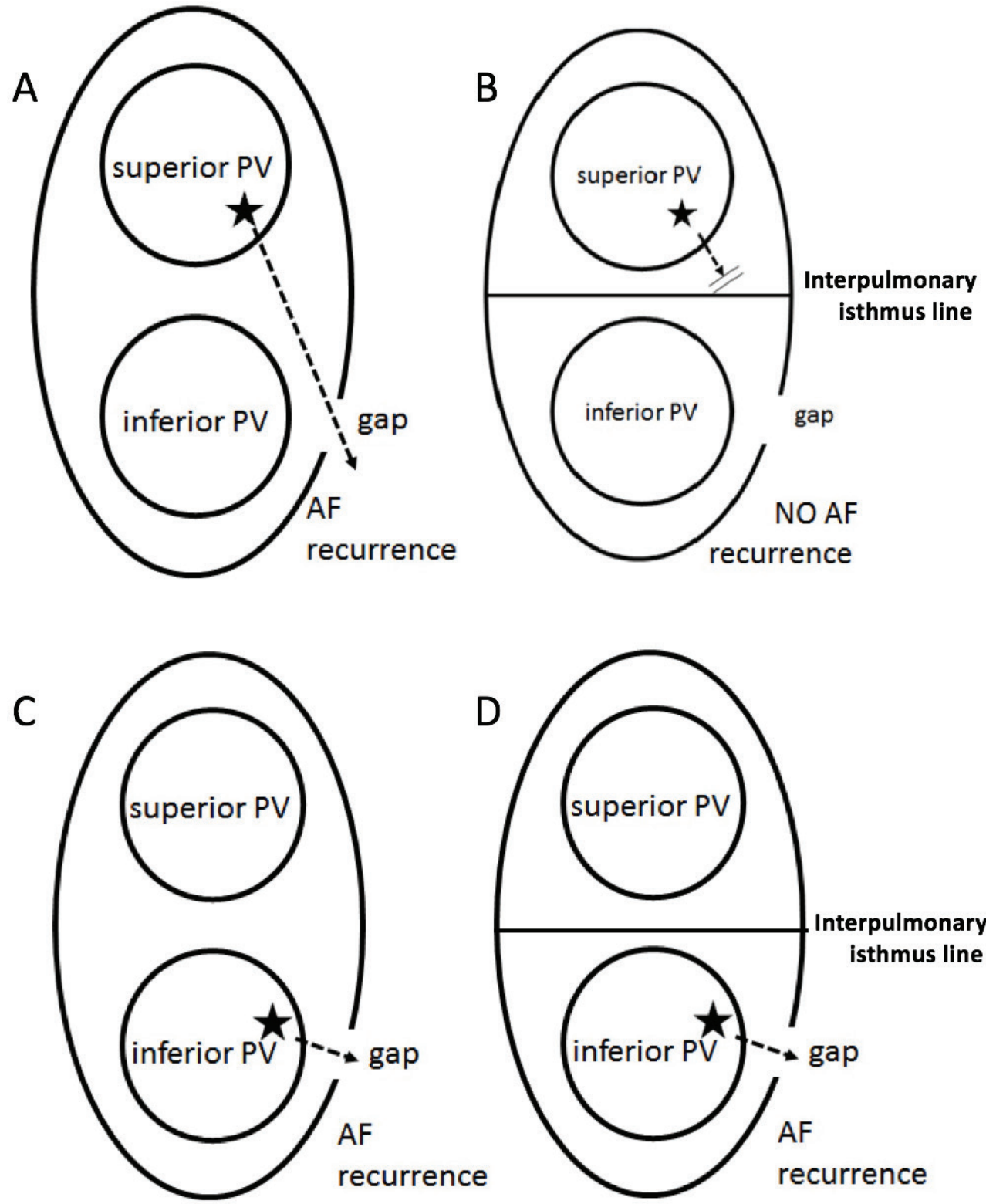

Figure 1 Hypothesis: When the reconduction site (gap) arises on the extensive encircling ablation line associated with the reconnection circuit from the opposite site trigger source (indicated by $\star$ ), penetrating the interpulmonary isthmus line to the reconduction site, AF is induced through this circuit (A). The interpulmonary isthmus line ablation can block this circuit, resulting in $\mathrm{AF}$ recurrence prevention (B). However, we can also hypothesise conversely that the reconnection circuit occurs between the trigger source and gap in the short circuit, (C) and the interpulmonary isthmus line ablation cannot block this circuit in this case (D). AF, atrial fibrillation; PV, pulmonary vein.

perpetuating $\mathrm{AF}^{7}$ Contribution of the intravenous ridge to PV reconnection has been reported. ${ }^{8}$ Moreover, the electrical connection between the superior and inferior PVs in patients with paroxysmal AF has been reported..$^{910}$ $\mathrm{PV}$ reconnection after EEPVI is observed at a significant rate, and this could cause $\mathrm{AF}$ recurrence.

The questions that the present study aimed to resolve are shown in figure 1. Two assumptions can be made. First, block electric current across the interpulmonary isthmus line is presumed to reduce AF recurrence rate when the reconduction site (gap) arises on the EEPVI ablation line associated with the reconnection circuit from the opposite site trigger source, crossing the interpulmonary isthmus line to the reconduction site, as shown in figure 1A,B. Second, in contrast, when gap exists in the EEPVI line and trigger source located on the same site, the reconnection circuit does not cross the isthmus line, resulting in interpulmonary isthmus line ablation not blocking the reconnection circuit (figure 1C,D). The present study intended to clarify which was significant. Furthermore, AF triggers can occur at a significant rate on the interpulmonary isthmus line. A study on double-Lasso technique that compared AF freedom after ablation between pulmonary vein isolation (PVI) and PVI with interpulmonary isthmus ablation reported no statistically significant differences in $\mathrm{AF}$ freedom between 
the two groups. ${ }^{11}$ The study included a relatively small number of patients and did not confirm bidirectional conduction block between the superior and inferior PVs. Further studies are required to confirm the effects of interpulmonary isthmus ablation on the AF recurrence rate. Consequently, we conducted a single-centre study comparing the AF recurrence rate between EEPVI with and without adjunctive interpulmonary isthmus line.

\section{METHODS}

We conducted a single-centre, randomised control study at Okayama Heart Clinic. We evaluated consecutive patients with symptomatic paroxysmal AF refractory to medical management who underwent their first ablation therapy. Exclusion criteria were as follows: patients requiring additional region or carina region ablation in addition to EEPVI to achieve PV isolation; patients with individual PV isolation of ipsilateral PVs despite EEPVI and patients with a common PV trunk due to lack of interpulmonary isthmus. Among 230 patients with paroxysmal AF who underwent PVI, 5, 10 and 15 patients requiring carina region ablation, with individual PVI and with a common PV trunk, respectively, were excluded. Consequently, 200 patients $(200 / 230$; 87\%) met the criteria and were enrolled in the present study. Patients were prospectively randomised to adjunctive interpulmonary isthmus ablation following the completion of EFPVI (group 1; $\mathrm{n}=100$ ) and EFPVI only (group 2; $\mathrm{n}=100$ ) between January 2012 and September 2012. The examination procedure complied with the rules of the Declaration of Helsinki, ${ }^{12}$ and the study was approved by the Institutional Ethics Committee for Human Research of Okayama Heart Clinic. Written informed consent was obtained from all patients.

\section{AF ablation}

Details of the present AF ablation procedure have been published elsewhere. ${ }^{13}$ Ablation was performed by using the double-Lasso technique. ${ }^{14}$ In brief, five venous accesses for electrical mapping and ablation were obtained as follows. Two standard electrophysiology catheters were positioned: a 4-F catheter (Japan Lifeline, Tokyo, Japan) at the His bundle region via a femoral vein and a $6-\mathrm{F}$ catheter in the coronary sinus via the right intrajugular vein. Three catheters were introduced in the LA by using the Brockenbrough technique, which requires two decapolar ring catheters (Japan Lifeline) and an open-irrigated, flexible-tip ablation catheter $\left(\mathrm{CoolFlex}^{\mathrm{TM}}\right.$, St Jude Medical, St Paul, Minnesota).

EEPVI was performed in all patients by using an open-irrigated ablation catheter inserted via the transseptal sheath with an electroanatomical integration mapping system (Ensite-NavX System, St Jude Medical). Irrigated radiofrequency (RF) energy was applied with a target temperature and infusion rate of $43^{\circ} \mathrm{C}$ and $8-15 \mathrm{~mL} /$ min, respectively. RF energy was delivered with a maximal power limit of $35 \mathrm{~W}$ and up to $60 \mathrm{~s}$ until decrease in the amplitude of the local bipolar atrial electrogram by $>80 \%$ or $<0.1 \mathrm{mV}$. The temperature of the oesophagus was continuously monitored by using a catheter with a temperature sensor (SensiTherm ${ }^{\mathrm{TM}}$, St Jude Medical) during ablation.

The EEPVI endpoint was defined as (1) the simultaneous PV potential elimination recorded by the two-ring catheters within the ipsilateral PVs and the lack of LA capture during intra-PV at least $30 \mathrm{~min}$ after isolation; and (2) no PV spike recurrence within all the PVs after the intravenous administration of 20-40 mg ATP during sinus rhythm or coronary sinus pacing.

When the PV potential still remained following the one-round, wide-area circumferential ablation around the ipsilateral veins, both PVs were mapped sequentially by the ring catheters to detect the earliest LA-PV conduction sites on the circumferential line and ablated these sites to isolate $\mathrm{PV}$.

RF energy in group 1 patients was supplied on the interpulmonary isthmus line, reaching the anterior and posterior portions of the encircling ablation line (figure 2). The endpoint of the interpulmonary isthmus ablation in group 1 was determined when bidirectional conduction block between the superior and inferior PVs was confirmed by PV pacing using ring catheters placed within the PVs with $20 \mathrm{mg}$ ATP injection (figure 3). After the completion of EEPVI and interpulmonary isthmus ablation in group 1 and EEPVI in group 2, $20 \mathrm{mg}$ ATP was rapidly administered intravenously to evaluate dormant conduction in PVs and interpulmonary isthmus. PVs and interpulmonary isthmus with conduction recovery after adenosine testing were re-ablated. Adenosine testing was repeated until dormant conduction disappeared.

\section{Postablation care and follow-up}

Anticoagulation therapy after the procedure was continued at least 3 months after the AF ablation in all groups. All patients were followed up monthly at least 90 days up to 36 months after the AF ablation at our centre. The initial follow-up was conducted 2 weeks after AF ablation. All previously ineffective antiarrhythmic drugs were withdrawn just after ablation. Surface ECG and transthoracic echocardiography were performed at our centre during follow-up. All patients had a telemetry ECG recorder (Omron, Kyoto, Japan) to document symptomatic arrhythmias or to transfer an ECG once per week if asymptomatic for 6 months. AF recurrence was defined as documented atrial arrhythmia (AF or atrial tachycardia) lasting $>30 \mathrm{~s}$. Enhanced CT was performed in all patients 3 months after the ablation to evaluate PV stenosis. PV stenosis was defined as $50 \%$ reduction of the luminal diameter of PV.

\section{Statistics}

Data were analysed using PASW Statistics V.17.0 (SPSS). Recurrence percentages were compared with $95 \%$ CIs of recurrence rate between the EEPVI with and without interpulmonary isthmus ablation. Student's t-test, $\chi^{2}$ 


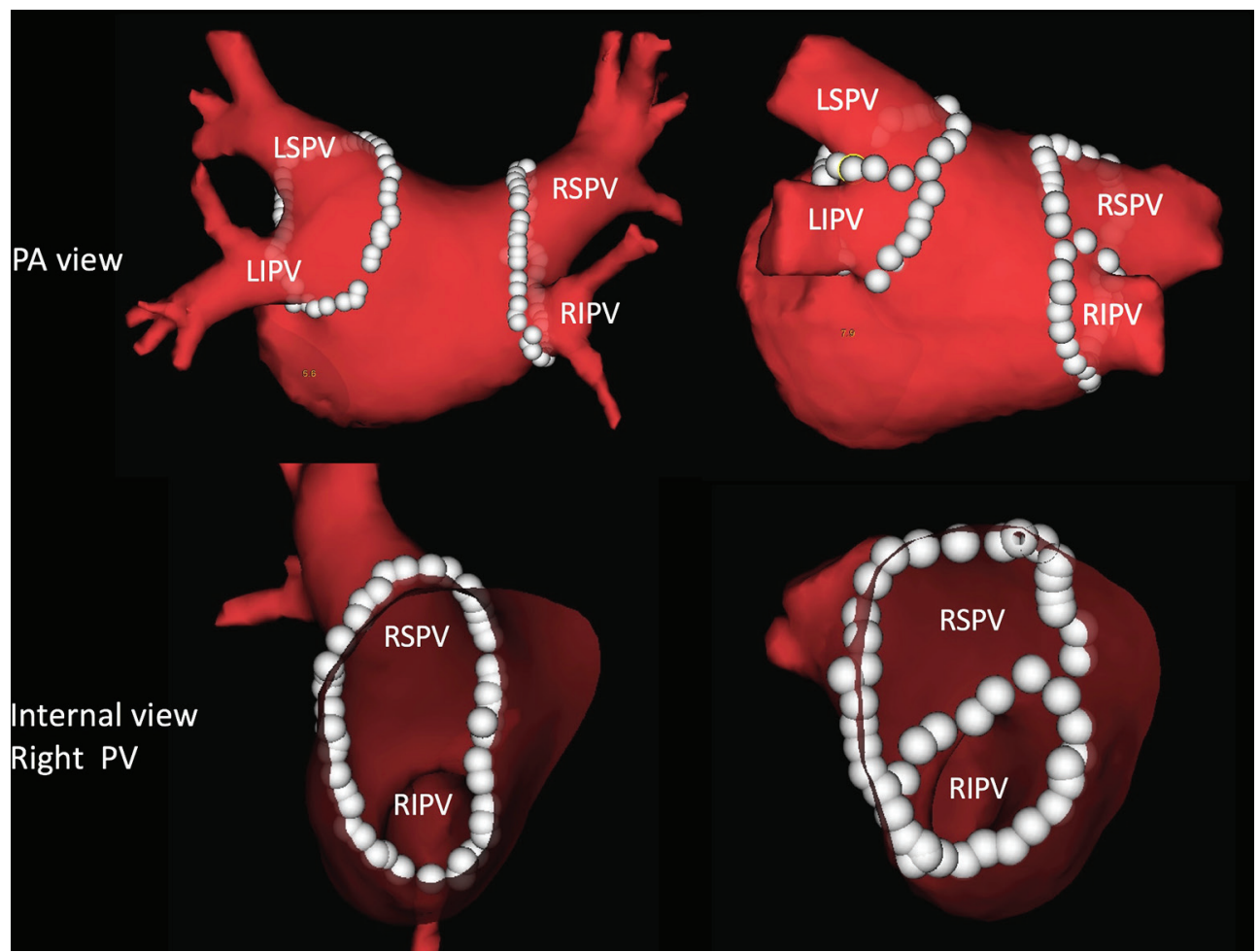

Figure 2 Comparison of extensive encircling pulmonary vein isolation with and without interpulmonary isthmus ablation on posteroanterior and internal views of the pulmonary veins. White dots represent ablation points. LSPV, left superior pulmonary vein; RSPV, right superior pulmonary vein; RIPV, right inferior pulmonary vein; LIPV, left inferior pulmonary vein; PA, posterioranterior; PV, pulmonary vein.

test with $2 \times 2$ tables and two-tailed test for continuous and categorical variables were used to compare the two groups. The Kaplan-Meier curve was used to compare the $\mathrm{AF}$ freedom between the two groups. Data are expressed as means $\pm 1 \mathrm{SD}$. Differences at $\mathrm{p}<0.05$ were considered significant. Power analysis was also performed to evaluate the sample size of the present study. The sample size of the present study had an $80 \%$ power at $\mathrm{p}=0.05$ to detect $16 \%-17 \%$ reduction in the $\mathrm{AF}$ recurrence rate from $30 \%$ to $35 \%$, which was estimated for the EPVI control group.

\section{RESULTS}

\section{Patient characteristics}

No significant differences were found in patients' background characteristics, such as age, sex and associated disorders, between groups 1 and 2 (table 1). No significant differences were seen in AF conditions, AF duration, $\mathrm{CHADS}_{2}$ score and $\mathrm{CHA}_{2} \mathrm{DS}_{2}$-VASc score between the two groups. Furthermore, echocardiographic parameters were not different between the two groups.

\section{Procedural parameters and ablation success}

The procedural parameters are summarised in table 2. The procedural parameters throughout the AF ablation, including procedural, fluoroscopic and RF energy supply times, in group 1 were significantly longer than those in group 2. All patients in both groups reached the EEPVI endpoint, and the initial success rate was, thus, not different between the two groups. Complete PVI was established without interpulmonary isthmus line ablation in group 2 patients. Interpulmonary isthmus ablation required approximately $10 \mathrm{~min}$ procedural time and resulted in $100 \%$ success rate.

\section{Complications}

Neither thromboembolic nor major bleeding complications were observed in groups 1 and 2 during the procedural and periprocedural periods (table 2). Minor bleeding complications rarely occurred in both groups. Late thromboembolic and bleeding complications after discharge were not observed in any patients during the follow-up period at least 90 days after the AF ablation. In summary, safety outcomes did not differ between the two groups. Similarly, no significant differences in safety outcomes were found between patients randomly assigned to groups 1 and 2. PV stenosis did not occur in groups 1 and 2.

\section{AF recurrence rate}

Group 1 showed 32\% (32/100; 95\% CI, $23.0 \%$ to $42.0 \%$ ) recurrence rate, which was similar to that of group 2 $(33 \%, 33 / 100,95 \%$ CI, $23.9 \%$ to $43.1 \%$ ) (table 3$)$. The $\chi^{2}$ test demonstrated no significant differences in per cent recurrence rate between the two groups, with probability value of 1.00. Figure 4 shows the AF-free Kaplan-Meier curve. The AF-free curve was not statistically different between the two groups, rather very similar $(\mathrm{p}=0.450)$. $\mathrm{PV}$ reconnection incidence was observed similarly in 
A

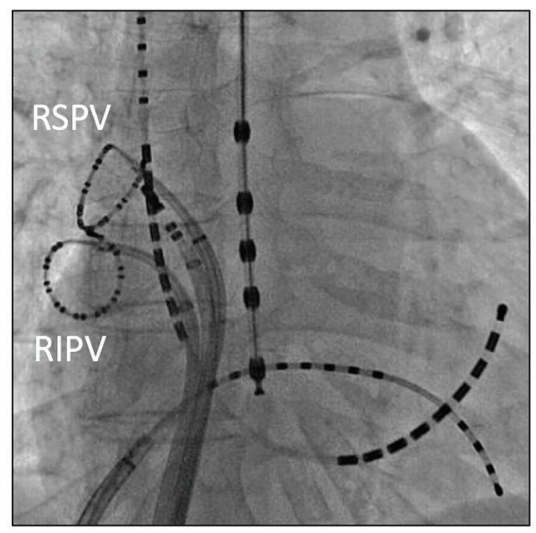

AP view
B

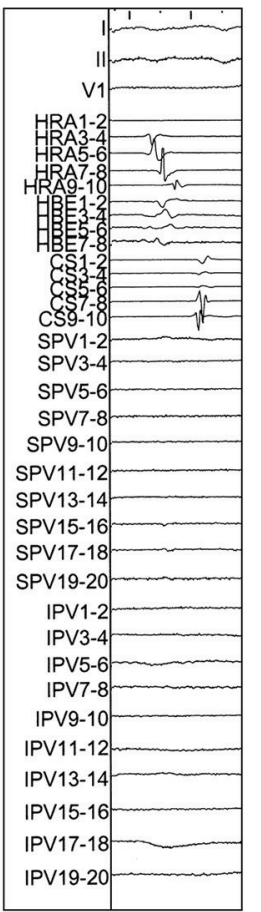

C

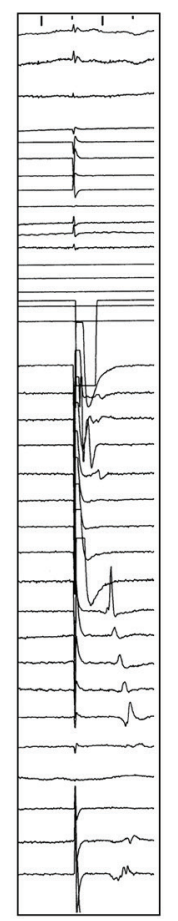

D $\quad E$

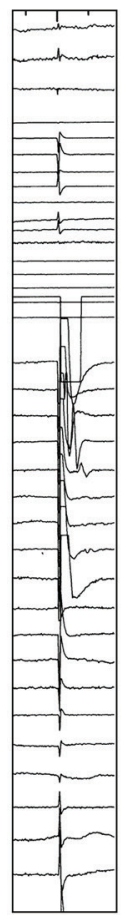

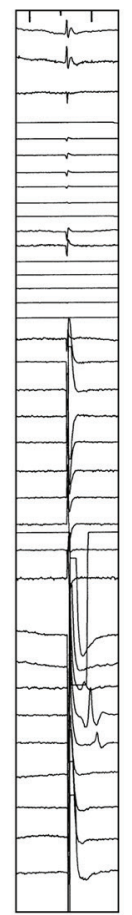

Figure 3 Representation figure of EEPVI with interpulmonary isthmus ablation. (A) Catheter positions during right PV isolation. (B) Intracardiac electrogram of the right superior (RSPV) and inferior (RIPV) pulmonary veins just after EEPVI. No PV potential was observed in both PVs. (C) Pacing at the superior PV by using a ring catheter. A local electrogram of the inferior PV was captured. (D) After interpulmonary isthmus ablation, local electrogram of the inferior PV was not captured despite pacing at the superior PV. (E) Local electrogram of the superior PV was not captured despite pacing at the inferior PV, hence the bidirectional block of the interpulmonary isthmus. AP, anterior-posterior; EEPVI, extensive encircling pulmonary vein isolation; PV, pulmonary vein.

both groups during the second session. The reconnection between the superior and inferior PVs, which is the reconnection through the interpulmonary isthmus line, was observed in $22 \%(6 / 27)$ of patients in group 1 who underwent a second session.

\section{DISCUSSION}

The present, prospective randomised, single-centre study revealed that $\mathrm{AF}$ recurrence rate was similar between EEPVI with and without adjunctive interpulmonary isthmus ablation, indicating that adjunctive interpulmonary isthmus ablation does not have obvious $\mathrm{AF}$ recurrence reduction effect.

The present EEPVI methods used, including electrophysiological monitoring and catheters, were almost identical with well-established methods. ${ }^{14}$ We applied the double-Lasso technique. The simultaneous records of ipsilateral PVs by two Lasso catheters detect the electrical interactions of the ipsilateral PVs and help identify conduction gaps in the EEPVI line. To make a complete EEPVI in the present study, the double-Lasso technique was suitable, and studies with application of this technique have been reported..$^{14} 15$ We did not use ablation catheter with contact force sensor (CF catheter). CF catheter has been reported not to improve AF recurrence, although $\mathrm{CF}$ catheter avoids both low and high CF. ${ }^{16}{ }^{17}$ Another randomised study demonstrated that $\mathrm{CF}$ catheter did not improve procedural and fluoroscopy times, or complication rates and 1-year success rates. ${ }^{18}$ In the present study, the fluoroscopic and procedural times of EEPVI in groups 1 and 2 were comparable or slightly superior to those in recent reports. ${ }^{1} 1920$ Interpulmonary isthmus ablation required approximately $10 \mathrm{~min}$. Similar results regarding adjunctive interpulmonary isthmus ablation not lengthening the procedural and fluoroscopic times have been reported. ${ }^{11}$ Furthermore, all patients reached the endpoint. Thus, application of the doubleLasso technique was appropriate. Furthermore, the use of open-irrigated ablation catheter, not CF catheter, was also satisfactory. Finally, these findings regarding procedural parameters indicated that our methods were reliable, and the results can be analysed.

In patients who underwent the second session in group 1, 88\% (21 of 27 patients) did not show electrical reconnection between the superior and inferior PVs, which demonstrated that electrical disconnection through the interpulmonary isthmus was durable. The results indicated that interpulmonary isthmus ablation maintained a non-conducting state in a considerable number of patients in group 1 . Thus, the AF recurrence rate must be different between the two groups when conduction block of the interpulmonary isthmus is hypothesised to play a significant role. In contrast 
to this theory, we found a similar AF recurrence rate. The results indicate that reconnection circuit across the isthmus line is ignorable. The finding proved that the interpulmonary isthmus did not play a significant role in $\mathrm{AF}$ recurrence.

We did not find a significant difference in the AF recurrence rate between patients with and without adjunctive interpulmonary isthmus line ablation, although intrapulmonary isthmus ablation was performed in an acceptable short time without significant complications, such as PV stenosis. Letsas $e t a l^{11}$ examined intrapulmonary isthmus ablation after complete PVI by using an ablation method similar to the present study, which is the double-Lasso technique, and did not show a decrease in AF recurrence rate. ${ }^{11}$ The study did not confirm the bidirectional conduction block between the superior and inferior PVs, whereas we did. In addition, the study examined a small number of patients (76 patients), including patients with chronic AF, and used the CARTO system. The study had lower patients' age and longer procedural time compared with the present study. The AF recurrence rate in the study seems slightly higher than that in the present study. Although these differences occur between their study and the present study, EEPVI ablation methods and additional interpulmonary isthmus line ablation methods were identical with the present study, and the results were also identical. The present study and the abovementioned

Table 1 Clinical characteristics, underlying disorders, arrhythmia conditions and echocardiographic parameters

\begin{tabular}{ll} 
Group 1 & Group 2 \\
\hline EEPVI + & \\
interpulmonary &
\end{tabular}

\begin{tabular}{|c|c|c|c|}
\hline & $n=100$ & $n=100$ & $p$ Value \\
\hline Age (years) & $63 \pm 11$ & $62 \pm 11$ & 0.75 \\
\hline Female & 34 & 28 & 0.44 \\
\hline \multicolumn{4}{|l|}{ Associated disease } \\
\hline Heart failure & 8 & 5 & 0.57 \\
\hline Hypertension & 41 & 53 & 0.09 \\
\hline Diabetes & 8 & 15 & 0.18 \\
\hline TIA/stroke & 8 & 5 & 0.57 \\
\hline \multicolumn{4}{|l|}{ AF conditions } \\
\hline $\begin{array}{l}\text { Duration of AF } \\
\text { (years) }\end{array}$ & $3 \pm 2$ & $3 \pm 2$ & 1 \\
\hline $\mathrm{CHADS}_{2}$ score & $0.8 \pm 0.8$ & $0.9 \pm 0.8$ & 0.81 \\
\hline $\begin{array}{l}\mathrm{CHA}_{2} \mathrm{DS}_{2}-\mathrm{VASC} \\
\text { score }\end{array}$ & $1.7 \pm 1.4$ & $1.6 \pm 1.2$ & 0.71 \\
\hline \multicolumn{4}{|l|}{$\begin{array}{l}\text { Echocardiographic } \\
\text { parameters }\end{array}$} \\
\hline LVEF (\%) & $65 \pm 7$ & $64 \pm 8$ & 0.83 \\
\hline LA diameter (mm) & $39 \pm 4$ & $38 \pm 5$ & 0.94 \\
\hline
\end{tabular}

Because the total number of patients in each group was 100, the number also indicates the percentage. Values are mean $\pm S D$. AF, atrial fibrillation; EEPVI, extensive encircling pulmonary vein isolation; LA, left atrium; LVEF, left ventricular ejection fraction; TIA, transient ischaemic attack.
Table 2 Comparison of procedural parameters and complications between adjunctive and non-adjunctive groups

\begin{tabular}{lll} 
Group 1 & Group 2 & \\
\hline $\begin{array}{l}\text { EEPVI + } \\
\text { interpulmonary } \\
\text { isthmus ablation } \\
n=100\end{array}$ & $\begin{array}{c} \\
\text { EEPVI } \\
n=100\end{array} \quad$ p Value
\end{tabular}

\begin{tabular}{|c|c|c|c|}
\hline \multicolumn{4}{|c|}{ Procedural parameters } \\
\hline $\begin{array}{l}\text { Procedural } \\
\text { time (min) }\end{array}$ & $111 \pm 23$ & $97 \pm 13$ & $<0.001$ \\
\hline $\begin{array}{l}\text { Fluoroscopic } \\
\text { time (min) }\end{array}$ & $32 \pm 9$ & $26 \pm 4$ & $<0.001$ \\
\hline $\begin{array}{l}\text { RF energy } \\
\text { supply time } \\
\text { (min) }\end{array}$ & $29 \pm 8$ & $26 \pm 9$ & $<0.001$ \\
\hline $\begin{array}{l}\text { PV isolation } \\
\text { success (\%) }\end{array}$ & 100 & 100 & 1 \\
\hline
\end{tabular}

Thromboembolic complications

(stroke/TIA, DVT or pulmonary embolism)

\begin{tabular}{|c|c|c|c|}
\hline & 0 & 0 & 1 \\
\hline \multicolumn{4}{|c|}{ Bleeding complications } \\
\hline \multicolumn{4}{|c|}{$\begin{array}{l}\text { Major bleeding complications } \\
\text { (periprocedural or late cardiac tamponade, retroperitoneal bleeding, } \\
\text { decreased haemoglobin level }>4 \mathrm{~g} / \mathrm{dL} \text {, blood transfusion required) }\end{array}$} \\
\hline & 0 & 0 & 1 \\
\hline \multicolumn{4}{|c|}{ Minor bleeding complications (n) } \\
\hline $\begin{array}{l}\text { Pericardial } \\
\text { effusion }\end{array}$ & 1 & 0 & 1 \\
\hline $\begin{array}{l}\text { Groin } \\
\text { haematoma }\end{array}$ & 2 & 2 & 1 \\
\hline Haematuria & 1 & 2 & 1 \\
\hline Other & 0 & 0 & 1 \\
\hline $\begin{array}{l}\text { Prolonged } \\
\text { hospitalisation }\end{array}$ & 0 & 0 & 1 \\
\hline \multicolumn{4}{|c|}{$\begin{array}{l}\text { Safety outcome (composite of bleeding and thromboembolic } \\
\text { complications) }\end{array}$} \\
\hline & $4(4 \%)$ & $4(4 \%)$ & 1 \\
\hline
\end{tabular}

Values are mean \pm SD.

EEPVI, extensive encircling pulmonary vein isolation; PV, pulmonary vein; RF, radiofrequency; TIA, transient ischaemic attack: DVT. deep vein thrombosis.

study show that no additive effects of adjunctive interpulmonary isthmus line ablation with EEPVI were found on AF recurrence.

Another multicentre study also found no incremental effects of additional intravenous ridge ablation on $\mathrm{AF}$ recurrences. $^{21}$ The ablation methods were different from those of the present study. The study did not use the double-Lasso technique and did not monitor the ipsilateral PV potentials during ablations. The results of the present study, thus, cannot directly compare with the reported results. The study found that PV potential faced the carina zone in $44 \%(51 / 117)$ of patients after circling PVI, and ablation on the carina site was required to 
Table 3 AF recurrence electrophysiological results during the second session and PV stenosis

\begin{tabular}{ll} 
Group 1 & Group 2 \\
\hline EEPVI + & \\
interpulmonary & \\
isthmus ablation & EEPVI
\end{tabular}

isthmus ablation EEPVI

\begin{tabular}{llll} 
& $\mathbf{n = 1 0 0}$ & $\mathbf{n}=\mathbf{1 0 0}$ & $\mathbf{p}$ Value \\
\hline $\begin{array}{l}\text { AF recurrence }(\mathrm{n}) \\
\text { Second session }\end{array}$ & 32 & 33 & 1 \\
$\begin{array}{l}\text { PV reconnection: } \\
\mathrm{n}(\% \text { of number of } \\
\text { second session) }\end{array}$ & $20(74 \%)$ & 25 & \\
$\begin{array}{l}\text { Non-PV foci: } \\
\mathrm{n}(\% \text { of number of }\end{array}$ & & & \\
second session) & $7(26 \%)$ & $5(20 \%)$ & 1 \\
$\begin{array}{l}\text { Reconnection } \\
\text { between superior } \\
\text { and inferior PVs: } \\
\mathrm{n} \text { (\% of number of } \\
\text { second session) }\end{array}$ & & & \\
PV stenosis $(\mathrm{n})$ & $0(22 \%)$ & & \\
\hline
\end{tabular}

AF, atrial fibrillation; EEPVI, extensive encircling pulmonary vein isolation: NA. not applicable: PV. pulmonary vein.

eliminate PV potential completely. ${ }^{21}$ In addition, several studies indicated that interpulmonary isthmus line ablation was required in establishing complete $\mathrm{PV}$ isolation. ${ }^{6}$ $22{ }^{23}$ Patients in the present study requiring ablation on the carina site were excluded to avoid interference with the comparison of any ablation on the carina site. Furthermore, the incidence of patients who required ablation on the carina site after EEPVI completion in the present study by using the double-Lasso technique was approximately $1 \%-2 \%$, which was considerably lower than the reported study. ${ }^{21}$ The study found a decrease in AF-free rate in patients who required carina site ablation to complete PV electrical isolation. Comparison of the present results with the reported results revealed that EEPVI measuring ipsilateral PV potential simultaneously by using two decapolar ring catheters to achieve complete PV isolation is important with respect to the AF-free rate.

\section{Study limitations}

First, although this study conducted a prospective random design, the number of patients examined was still relatively small. However, the clinical and demographic characteristics, ablation data and follow-up outcomes were not different between the two groups. Furthermore, both groups showed a similar AF-free rate and curve. Although the present study was statistically slightly underpowered as stated in the Statistics section, an increase in number seems highly to provide similar results. At least, the present results can provide data for meta-analyses. Second, the present study used a single-centre design. The results may not be acceptable widely. The methods were identical with those used in many studies and were not changed throughout the study period. The advantages of the single-centre study are that methods and operator technique were completely identical between the two groups. The single-centre design was not likely a major limitation of the study.

\section{CONCLUSION}

The results of our study showed similar AF recurrence rate between EEPVI with and without adjunctive interpulmonary isthmus ablation, indicating that interpulmonary isthmus ablation does not obviously decrease AF recurrence. This

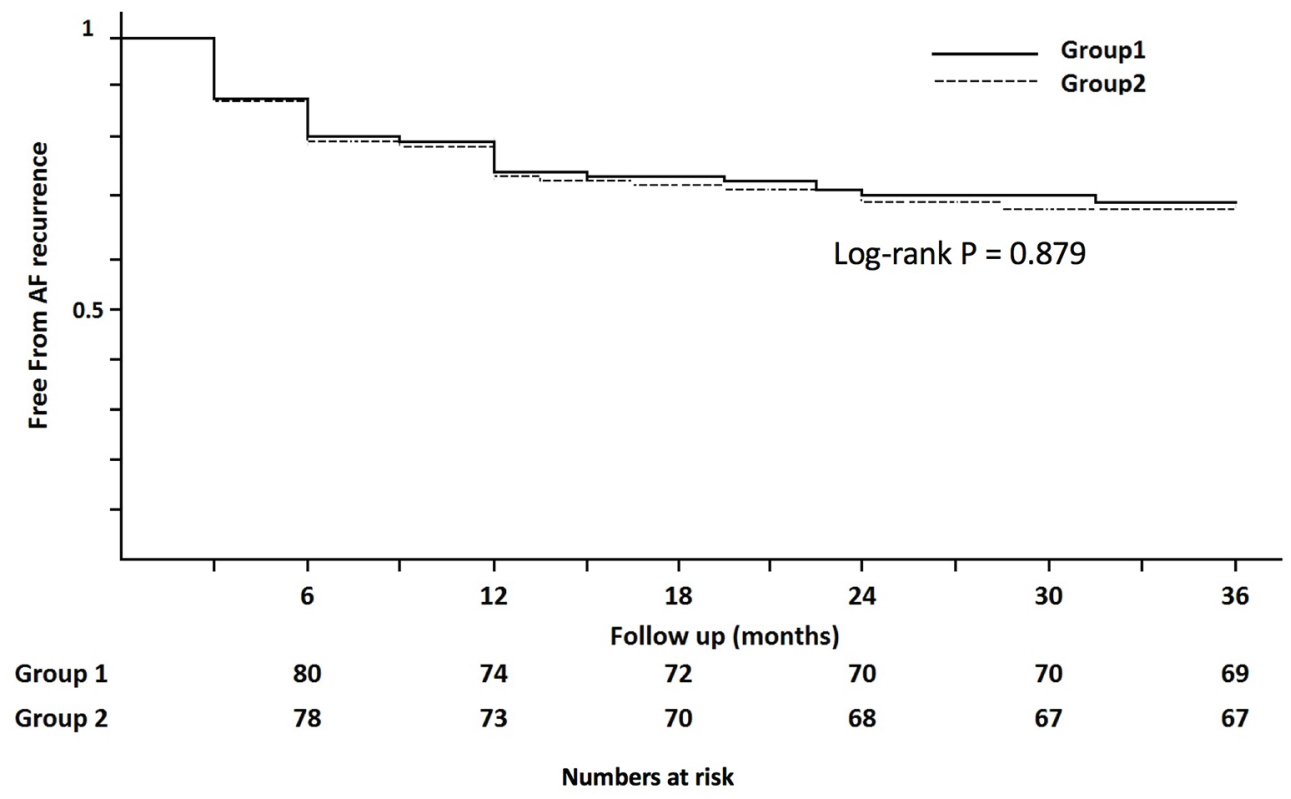

Figure 4 Cumulative freedom from AF without antiarrhythmic drug after a single procedure in study groups. Group 1: pulmonary vein isolation with interpulmonary isthmus ablation. Group 2: pulmonary vein isolation. AF, atrial fibrillation. 
study emphasises the importance of stable and complete $\mathrm{PV}$ isolation by EEPVI itself to reduce AF recurrence.

Contributors Conception and design of study: $\mathrm{SH}, \mathrm{HY}$; d rafting of the manuscript: $\mathrm{SH}, \mathrm{HY}, \mathrm{SK}$; r evising the manuscript critically for important intellectual content: HY, $\mathrm{SH}, \mathrm{SK}$; approval of the version of the manuscript to be published: $\mathrm{SH}, \mathrm{HY}, \mathrm{TM}, \mathrm{KH}$, HK, MM, SK, IK and SK.

Competing interests None declared.

Provenance and peer review Not commissioned; externally peer reviewed.

Open Access This is an Open Access article distributed in accordance with the Creative Commons Attribution Non Commercial (CC BY-NC 4.0) license, which permits others to distribute, remix, adapt, build upon this work noncommercially, and license their derivative works on different terms, provided the original work is properly cited and the use is non-commercial. See: http://creativecommons.org/ licenses/by-nc/4.0/

(c) Article author(s) (or their employer(s) unless otherwise stated in the text of the article) 2017. All rights reserved. No commercial use is permitted unless otherwise expressly granted.

\section{REFERENCES}

1. Calkins H, Brugada J, Packer DL, et al. HRS/EHRA/ECAS expert consensus statement on catheter and surgical ablation of atrial fibrillation: recommendations for personnel, policy, procedures and follow-up. Europace 2007;9:335-79.

2. Seshadri N, Marrouche N, Wilber D, et al. Pulmonary vein isolation for treatment of atrial fibrillation: recent updates. Pacing Clin Electrophysiol 2003;26:1636-40.

3. Haïssaguerre M, Jaïs $\mathrm{P}$, Shah DC, et al. Spontaneous initiation of atrial fibrillation by ectopic beats originating in the pulmonary veins. N Engl J Med 1998;339:659-66.

4. Lin WS, Tai CT, Hsieh MH, et al. Catheter ablation of paroxysmal atrial fibrillation initiated by non-pulmonary vein ectopy. Circulation 2003;107:3176-83.

5. Cappato R, Calkins H, Chen SA, et al. Updated worldwide survey on the methods, efficacy, and safety of catheter ablation for human atrial fibrillation. Circ Arrhythm Electrophysiol 2010;3:32-8.

6. Kistler PM, Ho SY, Rajappan K, et al. Electrophysiologic and anatomic characterization of sites resistant to electrical isolation during circumferential pulmonary vein ablation for atrial fibrillation: a prospective study. J Cardiovasc Electrophysiol 2007;18:1282-8.

7. Valles $\mathrm{E}, \mathrm{Fan} \mathrm{R}$, Roux JF, et al. Localization of atrial fibrillation triggers in patients undergoing pulmonary vein isolation: importance of the carina region. J Am Coll Cardiol 2008;52:1413-20.

8. Rajappan K, Kistler PM, Earley MJ, et al. Acute and chronic pulmonary vein reconnection after atrial fibrillation ablation: a prospective characterization of anatomical sites. Pacing Clin Electrophysiol 2008;31:1598-605.
9. Takigawa M, Kuwahara T, Takahashi A, et al. Simultaneous isolation of superior and inferior pulmonary veins on both the left and right sides could yield better outcomes in patients with paroxysmal atrial fibrillation. Europace 2015;17:732-40.

10. Takahashi Y, lesaka Y, Takahashi A, et al. Electrical connection between left superior and inferior pulmonary veins in a patient with paroxysmal atrial fibrillation. J Cardiovasc Electrophysiol 2002;13:490-2.

11. Letsas KP, Efremidis M, Vlachos K, et al. The impact of catheter ablation in the interpulmonary isthmus on atrial fibrillation ablation outcomes: a randomized study. J Cardiovasc Electrophysiol 2014:25:709-13.

12. World Medical Association Declaration of Helsinki. Ethical principles for medical research involving human subjects. JAMA 2013;310:2191-4

13. Yamaji $\mathrm{H}$, Murakami T, Hina $\mathrm{K}$, et al. Usefulness of dabigatran etexilate as periprocedural anticoagulation therapy for atrial fibrillation ablation. Clin Drug Investig 2013;33:409-18.

14. Ouyang F, Bänsch D, Ernst S, et al. Complete isolation of left atrium surrounding the pulmonary veins: new insights from the double-Lasso technique in paroxysmal atrial fibrillation. Circulation 2004;110:2090-6.

15. Ouyang F, Ernst S, Chun J, et al. Electrophysiological findings during ablation of persistent atrial fibrillation with electroanatomic mapping and double lasso catheter technique. Circulation 2005;112:3038-48.

16. Deubner N, Greiss H, Akkaya E, et al. Clinical experience with contact-force and flexible-tip ablation catheter designs. J Interv Card Electrophysiol 2016;47:75-82.

17. Stabile G, Solimene F, Calò L, et al. Catheter-tissue contact force values do not impact mid-term clinical outcome following pulmonary vein isolation in patients with paroxysmal atrial fibrillation. J Interv Card Electrophysiol 2015;42:21-6.

18. Ullah W, McLean A, Tayebjee MH, et al; UK Multicentre Trials Group**. Randomized trial comparing pulmonary vein isolation using the smarttouch catheter with or without real-time contact force data. Heart Rhythm 2016;13:1761-7.

19. Rostock T, Steven D, Hoffmann B, et al. Chronic atrial fibrillation is a biatrial arrhythmia: data from catheter ablation of chronic atrial fibrillation aiming arrhythmia termination using a sequential ablation approach. Circ Arrhythm Electrophysiol 2008;1:344-53.

20. Schmidt B, Tilz RR, Neven K, et al. Remote robotic navigation and electroanatomical mapping for ablation of atrial fibrillation: considerations for navigation and impact on procedural outcome. Circ Arrhythm Electrophysiol 2009;2:120-8

21. McLellan AJ, Ling LH, Azzopardi S, et al. A minimal or maximal ablation strategy to achieve pulmonary vein isolation for paroxysmal atrial fibrillation: a prospective multi-centre randomized controlled trial (the minimax study). Eur Heart $J$ 2015;36:1812-21.

22. Udyavar AR, Chang SL, Tai CT, et al. The important role of pulmonary vein carina ablation as an adjunct to circumferential pulmonary vein isolation. J Cardiovasc Electrophysiol 2008;19:593-8.

23. Kumagai K, Naito S, Nakamura K, et al. ATP-induced dormant pulmonary veins originating from the carina region after circumferential pulmonary vein isolation of atrial fibrillation. J Cardiovasc Electrophysiol 2010;21:494-500. 